\title{
Diverse Synaptic Mechanisms Generate Direction Selectivity in the Rabbit Retina
}

\author{
W. Rowland Taylor ${ }^{1}$ and David I. Vaney ${ }^{2}$ \\ 1John Curtin School of Medical Research and Centre for Visual Sciences, Australian National University, Canberra, 2601 \\ ACT, Australia, and 'Vision, Touch and Hearing Research Centre, School of Biomedical Sciences, The University of \\ Queensland, Brisbane, QLD 4072, Australia
}

The synaptic conductance of the On-Off direction-selective ganglion cells was measured during visual stimulation to determine whether the direction selectivity is a property of the circuitry presynaptic to the ganglion cells or is generated by postsynaptic interaction of excitatory and inhibitory inputs. Three synaptic asymmetries were identified that contribute to the generation of direction-selective responses: (1) a presynaptic mechanism producing stronger excitation in the preferred direction, (2) a presynaptic mechanism producing stronger inhibition in the opposite direction, and (3) postsynaptic interac-

The direction-selective ganglion cells (DSGCs) in the rabbit retina are a model system for investigating neural computation (Vaney et al., 2001). These cells respond strongly to an image moving in a preferred direction but only weakly to an image moving in the opposite "null" direction. The foundation for understanding the cellular mechanisms of direction selectivity in vertebrates was laid by Barlow and Levick (1965), whose extracellular recordings from DSGCs indicated that direction selectivity was mediated primarily by inhibition activated by nulldirection image motion. Strong support for the inhibitory model was provided by subsequent pharmacological experiments, which showed that $\mathrm{GABA}_{\mathrm{A}}$-receptor antagonists abolish direction selectivity (Wyatt and Daw, 1976; Ariel and Daw, 1982; Kittila and Massey, 1997). However, these extracellular recording experiments provided no information about whether the inhibition acted directly on the DSGC or presynaptically on the excitatory interneurons.

Torre and Poggio (1978) proposed a postsynaptic model in which DSGCs receive an inhibitory input that is spatially offset relative to the excitatory input; moreover, the inhibition is nondirectional, being activated equally well by image motion in the preferred and null directions. During null-direction motion, the spatial offset means that delayed inhibitory synapses at locations ahead of the stimulus are activated and veto the excitation as the stimulus sweeps across the receptive field. For preferreddirection motion, the inhibition trails behind the stimulus and thus arrives too late to veto the excitation. For this model to work, the inhibition must act locally within the dendritic arbor of the

Received Feb. 20, 2002; revised June 10, 2002; accepted June 11, 2002.

This work was supported by National Health and Medical Research Council grants to D.I.V. and W.R.T. We thank an anonymous reviewer and Dr. Lyle Graham for their constructive criticisms that allowed us to improve this manuscript.

Correspondence should be addressed to Dr. W. Rowland Taylor, Neurological Sciences Institute, Oregon Health and Sciences University-West Campus, 505 Northwest 185th Avenue, Beaverton, OR 97006. E-mail: taylorw@ohsu.edu.

Copyright (C) 2002 Society for Neuroscience $0270-6474 / 02 / 227712-09 \$ 15.00 / 0$ tion of excitation with spatially offset inhibition. Although the on- and off-responses showed the same directional tuning, the off-response was generated by all three mechanisms, whereas the on-response was generated primarily by the two presynaptic mechanisms. The results indicate that, within a single neuron, different strategies are used within distinct dendritic arbors to accomplish the same neural computation.

Key words: direction selectivity; ganglion cells; synaptic conductance; inhibition; excitation; dendritic integration; on- and off-pathways; rabbit retina

DSGC. This occurs when the inhibitory reversal potential is at, or close to, the resting potential of the cell, and therefore the inhibitory input does not polarize the cell but introduces a local increase in the membrane conductance, which reduces or "shunts" nearby excitatory inputs. The inhibition that generates direction selectivity appears to be divisive rather than subtractive (Amthor and Grzywacz, 1991), which is consistent with, but not indicative of, a postsynaptic mechanism.

Such a postsynaptic model contrasts with a presynaptic model in which the inputs to the DSGC are themselves directionally selective. There are two basic versions of the presynaptic model: (1) the excitatory inputs to the DSGC are larger in the preferred direction, and (2) the inhibitory inputs to the DSGC are larger in the null direction. These two versions could also coexist, with both the excitation and inhibition being directional. Moreover, presynaptic and postsynaptic mechanisms are not mutually exclusive and could conceivably be combined within a single ganglion cell.

A study of rabbit DSGCs by Taylor et al. (2000) showed that inhibition acts directly on the ganglion cells and that the synaptic inputs were balanced in the preferred and null directions. These results could be explained parsimoniously if the inhibitory input was nondirectional, thus favoring a postsynaptic mechanism of direction selectivity. A recent study of turtle DSGCs by BorgGraham (2001) measured synaptic conductance at different time points and then estimated the excitatory and inhibitory components. These experiments supported a presynaptic model by showing that the DSGCs receive excitatory inputs that are already directional, although the inhibitory inputs did not contribute to generating the direction selectivity.

Thus, the findings and conclusions from the turtle study are almost diametrically opposed to those from the rabbit study, leading us to undertake a similar conductance analysis of the light-evoked responses of DSGCs in the rabbit retina. 


\section{MATERIALS AND METHODS}

Animals and patch recording. The experiments comply with the Australian Capital Territory Animal Welfare Act (1992) and were approved by the Animal Experimentation Ethics Committee of the Australian National University. Dark-adapted, New Zealand White rabbits were surgically anesthetized, and the right eye was removed under dim-red illumination. The animal was then killed by anesthetic overdose. All subsequent manipulations were performed under infrared illumination. The front of the eye was removed, the eyecup was transected just above the visual streak, and the dorsal piece was discarded.

The retina was dissected from the sclera, and a $10 \times 10 \mathrm{~mm}$ section of central retina was adhered, photoreceptor-side down, to a coverslip coated with CellTak (BD Sciences, Bedford, MA). The whole-mount retina preparation was placed in a recording chamber $(\sim 0.5 \mathrm{ml}$ volume $)$ and perfused continually $(\sim 2 \mathrm{ml} / \mathrm{min})$ with oxygenated bicarbonatebuffered Ames medium, $\mathrm{pH} 7.4$, at $33-37^{\circ} \mathrm{C}$. Patch electrodes were pulled from borosilicate glass to have a final resistance of 4-8 $\mathrm{M} \Omega$.

For extracellular recording, the electrodes were filled with the Ames medium. For intracellular recording, the electrodes were filled with the following electrolytes: $110 \mathrm{~mm}$ Cs-gluconate or CsCl, $10 \mathrm{~mm} \mathrm{NaCl}, 5 \mathrm{~mm}$ Na-HEPES, $1 \mathrm{~mm}$ Cs-EGTA, $1 \mathrm{~mm}$ Na-ATP, $0.1 \mathrm{~mm}$ Na-GTP, and 10 mM QX-314 (Sigma-Aldrich). Cesium was used in place of potassium to block voltage-gated potassium currents and thereby improve the quality of the voltage clamp at positive potentials. The QX-314 was included to block voltage-dependent sodium channels and abolished all spiking activity within 1-2 min of establishing the whole-cell configuration. The liquid junction potential of $10 \mathrm{mV}$ was subtracted from all voltages when the intracellular solution contained Cs-gluconate.

Ganglion cells with a medium-large soma and a crescent-shaped nucleus were targeted as potential DSGCs (Vaney, 1994). The extracellular electrode was applied to the soma under visual control, after a broken patch-electrode was used to make a small hole in the overlying inner limiting membrane. After establishing that the ganglion cell was an On-Off DSGC and determining its preferred direction, the extracellular recording electrode was removed, and an intracellular electrode was applied to the same cell, again under visual control.

Light stimuli. Light stimuli were generated on a Barco Systems monitor (refresh rate, $72 \mathrm{~Hz}$ ) and focused onto the photoreceptor outer segments through a $40 \times$ [numerical aperture (NA) 0.75] or $20 \times(\mathrm{NA} 0.35)$ Olympus water-immersion objective. The stimulus contrast, defined as $C=\left(L_{\max }-L_{\min }\right) /\left(L_{\max }+L_{\min }\right)$, was set between 0.3 and 1.0. The standard moving stimulus comprised a light or dark bar, moving along its long axis at $800-1200 \mu \mathrm{m} / \mathrm{sec}$ on the retina. All light stimuli were centered with respect to the tip of the recording electrode and thus also with the soma of the ganglion cell. Relative timing of the responses in the preferred and null directions is made directly from the recorded responses without correction for possible spatial offset of the receptive fields. Justification for this is presented in the results. The width of the bar was $250 \mu \mathrm{m}$, and its length was set to achieve good temporal separation of the leading- and trailing-edge responses. These responses are clear in the Figures, but more often a distinction will be made between the on-response and the off-response. Because the stimulus could be either a light or dark bar, the leading-edge response could be either an on-response or an off-response.

Conductance analysis. Conductance analysis methods were similar to those described by Borg-Graham (2001). It was assumed that the lightevoked synaptic inputs comprised two components: an excitatory component because of activation of nonselective cation channels having a reversal potential, $V_{e}=0 \mathrm{mV}$, and an inhibitory component with a reversal potential, $V_{i}^{e}$, at the chloride equilibrium potential of approximately $-65 \mathrm{mV}$. The synaptic currents resulting from each of these components obey Ohm's law such that $I_{\mathrm{e}}=g_{\mathrm{e}}(t)\left(V-V_{\mathrm{e}}\right)$, and $I_{\mathrm{i}}=$ $g_{\mathrm{i}}(t)\left(V-V_{\mathrm{i}}\right)$, where the inhibitory and excitatory conductances, $g_{i}(t)$ and $g_{e}(t)$, respectively, are both functions of time. We assume that the DSGC is isopotential, so that the synaptic currents sum linearly, and the total light-evoked synaptic current is:

$$
I_{\mathrm{T}}=g_{\mathrm{T}}(t)\left(V-V_{\mathrm{r}}(t)\right)
$$

where $g_{T}(t)=g_{\mathrm{e}}(t)+g_{\mathrm{i}}(t)$, and $V_{r}(t)$ is the observed reversal potential. Thus, $V_{r}(t)$ is the weighted sum of $V_{e}$ and $V_{i}$ such that:

$$
V_{\mathrm{r}}(t)=\frac{g_{\mathrm{e}}(t)}{g_{\mathrm{T}}(t)} V_{\mathrm{e}}+\frac{\mathrm{g}_{\mathrm{i}}(t)}{g_{\mathrm{T}}(t)} V_{\mathrm{i}}
$$

Inspection of Equation 2 reveals that if the time courses of $g_{i}$ and $g_{e}$ are equal (can be superimposed under scalar multiplication), then $V_{r}(t)=$ constant (see Fig. 2). Equation 2 can be rearranged to separate the excitatory and inhibitory components from $g_{T}(t)$ and $V_{r}(t)$, where:

$$
\begin{aligned}
g_{\mathrm{i}}(t) & =\frac{g_{\mathrm{T}}(t)\left(V_{\mathrm{r}}(t)-V_{\mathrm{e}}\right)}{V_{\mathrm{i}}-\mathrm{V}_{\mathrm{e}}} \\
g_{\mathrm{e}}(t) & =\frac{g_{\mathrm{T}}(t)\left(V_{\mathrm{r}}(t)-V_{\mathrm{i}}\right)}{V_{\mathrm{e}}-V_{\mathrm{i}}} .
\end{aligned}
$$

Separation of the components required assigning values to the reversal potentials for excitation and inhibition, $V_{e}$ and $V_{i}$, in Equations 3 and 4. Application of these equations to the biophysical system studied here places constraints on $V_{e}$ and $V_{i}$. First, because of the ionic selectivity of the channels and the ionic gradients in the neurons, $V_{i}<V_{e}$. This will also be true when the high-chloride intracellular solutions are used, because it is unlikely that the chloride concentration within the dendrites will attain the chloride concentration in the recording electrode, because of chloride extrusion across the dendritic membranes (Vardi et al., 2000). A second constraint arises because $g_{i}, g_{e} \geq 0$, which means that $V_{i} \leq$ $V_{r} \leq V_{e}$. A third constraint was on the inner limits of $V_{e}$ and $V_{i}$, such that $V_{e} \geq 0 \mathrm{mV}$ and $V_{i} \leq-65 \mathrm{mV}$. These inner limit values for $V_{e}$ and $V_{i}$ are expected if there were no voltage clamp errors. In practice, if $V_{r}$ reached levels $>0 \mathrm{mV}$, then $V_{e}$ was set to equal or just exceed the most positive excursion of $V_{r}$. Similarly, if $V_{r}$ dipped below $-65 \mathrm{mV}$, then $V_{i}$ was set to equal or be just below the most negative $V_{r}$ value. $V_{e}$ and $V_{i}$ were assigned values to the nearest $5 \mathrm{mV}$. For high-chloride intracellular solutions, $V_{i}$ was allowed to be more positive than $-65 \mathrm{mV}$ but was only set positive enough to ensure that $g_{i}, g_{e} \geq 0$ at all times.

Errors in the assignment of the reversal potentials will result in quantitative errors in the estimates of the conductance ratios. The cell illustrated in Figure 2 provides a convenient example. If we were to set $V_{i}$ to $-45 \mathrm{mV}$, approximating the observed $V_{r}$ during null-direction motion, then according to Equation 4, in the null direction $g_{e} \approx 0$. Because $V_{r}$ approaches $0 \mathrm{mV}$ in the preferred direction, indicating $g_{e}>0$, an almost infinite preferred-null conductance ratio for $g_{e}$ would be predicted. Therefore, the constraint that $V_{i}$ not exceed $-65 \mathrm{mV}$ is conservative, but it is important to note that the qualitative result is unchanged: $g_{e}$ remains directional.

The synaptic conductance and reversal potential were evaluated as follows. Synaptic currents were elicited by moving the stimulus in the preferred and null directions at a series of holding potentials starting at $-100 \mathrm{mV}$ and incrementing by $15 \mathrm{mV}$ to $+20 \mathrm{mV}$. From these data, current-voltage $(I-V)$ relations of the net light-evoked current were constructed. The resting membrane $I-V$ relation was estimated by measuring the average membrane current level over a $0.15 \mathrm{sec}$ interval at the start of each voltage pulse, before the light-evoked response. This resting $I-V$ relation was subtracted from $I-V$ relations constructed every $10 \mathrm{msec}$ for the duration of the synaptic responses to produce the net light-evoked $I-V$ relations. The slope $\left(g_{T}\right)$ and intercept $\left(V_{r}\right)$ were determined from the light-evoked $I-V$ relation at each time point, thus producing discrete estimates of the functions $g_{T}(t)$ and $V_{r}(t)$. An uppercase "G" with corresponding subscript denotes the integrals of the conductance functions. In most cells, multiple $I-V$ runs were analyzed to ensure that the responses were reproducible.

Because thousands of $I-V$ relations were generated, it was not practical to fit and inspect the $I-V$ manually at each time point. Therefore, an automated routine was implemented using Igor Pro to perform linear regression on the $I-V$ relations (Wavemetrics, Lake Oswego, OR). In some cells, the $I-V$ relations at the most negative and most positive voltages were sublinear, tending to bend toward the voltage axis. At negative potentials, the nonlinearity could reflect activation of NMDA receptors, which are known to be expressed in these neurons (Kittila and Massey, 1997). The negative rectification often disappeared during the recording period. This phenomenon will not be examined further here. Because of the sublinear behavior at the extremes of the $I-V$ relations, linear regression over the full voltage range will not accurately represent the true synaptic conductance or reversal potential. To obviate this difficulty, we used two alternative procedures. In the first, $I-V$ relations were fit over a fixed voltage range from -70 to $-10 \mathrm{mV}$. In the second procedure, we allowed the automated routine to "choose" the best fit. All possible subsets of five to six contiguous points (from a possible nine spanning the voltage range -100 to $+20 \mathrm{mV}$ ) were fitted to each $I-V$, and the fit that produced the largest value for $g_{T}$ was accepted. Using the 


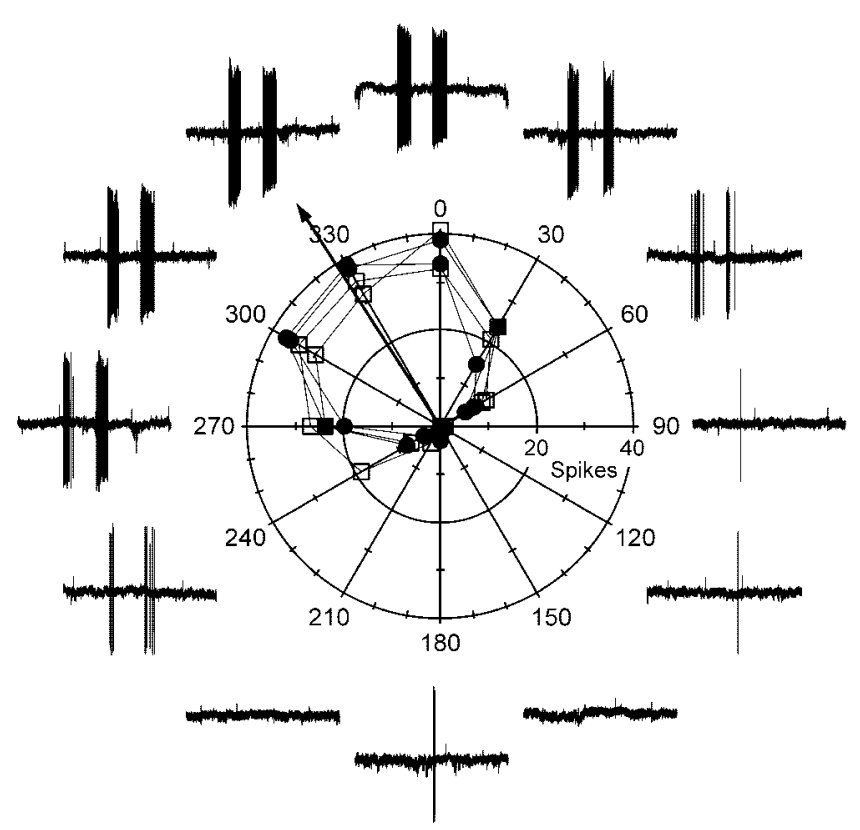

Figure 1. Determination of the preferred-null axis. The distance from the origin represents the total number of spikes produced by the leading edge (@) or trailing edge $(\square)$ of a single stimulus. The surrounding traces show the extracellular responses for the corresponding directions. The first burst of spikes, produced by the leading edge of the stimulus bar, is the off-response, because the moving bar was darker than the background ( 0.7 contrast). The second burst of spikes, produced by the trailing edge, is the on-response. The preferred directions for the on- and off-responses, calculated from the vector sum of the data points, were indistinguishable in this cell, and therefore they are marked by a single arrow, the length of which has been truncated; the lengths of the vector sums were 117 spikes for the on-responses and 120 spikes for the off-responses.

largest value for $g_{T}$ introduced a slight systematic bias toward larger conductances but avoided larger errors that would be introduced by the nonlinearities at the extremes of the $I-V$ relations. The peak conductance estimates were essentially identical, although, as expected, the second method produced slightly larger conductance estimates at low levels when the signal became noisy.

A direction-selectivity index, $D$, was calculated as a measure of the directional tuning. Extracellular action potentials were recorded in each of 8 or 12 stimulus directions, equally spanning $360^{\circ}$ at 45 or $30^{\circ}$ intervals. $D$ was defined for the action potential discharges as:

$$
D=\left|\frac{\sum \vec{v}_{\mathrm{i}}}{\sum r_{\mathrm{i}}}\right|,
$$

where $\nu_{\mathrm{i}}$ are vectors pointing in the direction of the stimulus and having length, $r_{i}$, equal to the number of spikes recorded during that stimulus. $D$ can range from 0 , when the responses are equal in all stimulus directions, to 1 , when a response is obtained only for a single stimulus direction. Thus, values for $D$ approaching 1 indicate asymmetric responses over a small range of angles and therefore sharper directional tuning.

\section{RESULTS}

\section{Directional tuning}

The preferred-null axis of each cell was established from extracellular recordings. A stimulus bar was swept across the receptive field in 12 directions, spaced at $30^{\circ}$ intervals (Fig. 1). The stimulus direction that generated the most action potentials was designated as the preferred direction, and the opposite direction was designated as the null direction. This preferred-null axis was used for all further stimuli during subsequent patch-clamp recordings. The preferred direction was calculated more precisely, during later analysis, from the vector sum of the leading- and trailing-edge spikes (Fig. 1, arrow). In a sample of 10 cells, the average absolute difference between the estimated preferred-null axis and the calculated axis was $12 \pm 8^{\circ}$, with the largest difference being $28^{\circ}$. The calculated preferred directions for the onand off-responses agreed very closely, differing by only $0.3 \pm 11^{\circ}$ in a group of 11 cells. Directional tuning, evaluated as defined by Equation 5 in Materials and Methods, was the same for the onand off-responses (Table 1).

\section{Synaptic current-voltage relations}

After the DSGC was patched, the membrane potential was stepped to a range of levels, and the visual stimulus was swept in opposite directions along the preferred-null axis. At negative potentials, the peak inward currents in the preferred and null directions were coincident both for the on- and off-responses, suggesting that the receptive field of the DSGC was well centered with respect to the stimulus (Fig. $2 A, B$ ). Therefore, comparisons of response timings in preferred and null directions are made directly from the records.

The sets of current records for the preferred and null directions (Fig. $2 A, B$ ) show systematic changes in the time course of the responses as a function of the holding potential, reflecting the changing balance of excitation and inhibition that occurs as the stimulus sweeps across the receptive field. In particular, at positive holding potentials, where the inhibitory inputs are more evident, the pronounced differences in the current wave-forms for preferred and null directions support the hypothesis that postsynaptic inhibition is important for generating direction selectivity (Taylor et al., 2000).

A quantitative analysis of these responses was performed by generating $I-V$ relations of the net light-evoked current every 10 msec for the duration of the visual responses. The $I-V$ relations were essentially linear over much of the voltage range (Fig. 2E), and the conductance was estimated by fitting a straight line to each $I-V$ plot. The fitted lines provided two parameters at each time point: the intercept, giving an estimate of the synaptic reversal potential $V_{r}$ (Eq. 1; Fig. $2 C, F, G$ ), and the slope, giving the size of the light-evoked conductance $g_{T}$ (Fig. $2 D, H$ ).

\section{Synaptic reversal potential}

The trajectory of the synaptic reversal potential was similar in every DSGC. In the preferred direction, $V_{r}$ ramped up rapidly toward $0 \mathrm{mV}$ during the early phase of the response, to both the leading edge (on-response) and the trailing edge (off-response) of a positive-contrast stimulus (Fig. $2 C$, blue line). $V_{r}$ then ramped down during the late phase of the response, typically ending around $-50 \mathrm{mV}$. The occurrence of the positive reversal potential correlated precisely with the timing of extracellular spikes recorded before the intracellular recording, as shown for another cell (Fig. 2F).

In the null direction, $V_{r}$ generally remained negative, consistent with the dearth of spikes in the extracellular recordings. During the late phase of the off-response, however, $V_{r}$ consistently ramped toward more positive values, producing a crossover in the reversal potential trajectories. When a positive-contrast stimulus was used, the crossover occurred during the late phase of the trailing-edge response (Fig. 2C,F, $X$ arrow). When a negative-contrast stimulus was used, the crossover occurred during the late phase of the leading-edge response (Fig. 2G, X arrow). The presence or absence of the crossover was robust enough to allow the sign of the contrast edge to be reliably determined by inspection of the $V_{r}$ records. Such crossover was never observed in the reversal potential trajectories 
Table 1. Directional index, conductance ratios, timing differences, and spatial offsets

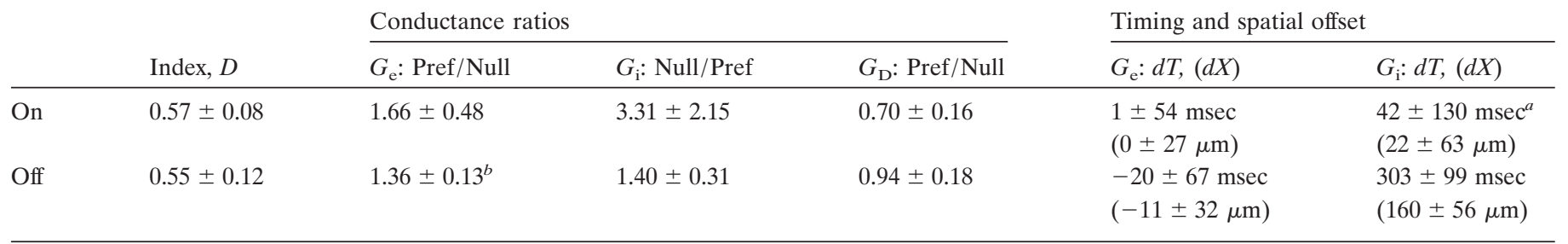

Values are quoted as the mean \pm SD for the 16 cells. Pref, Preferred direction; Null, null direction; Index, the directional index, $D$, is defined by Equation 5; Conductance ratios, average ratios of the integrated conductance for the on- and off-responses. The integrals $G_{\mathrm{e}}$ and $G_{\mathrm{i}}$ were calculated for the 16 cells over the limits shown by the solid lines under the records in Figure 4. $G_{\mathrm{D}}=G_{\mathrm{e}}+G_{\mathrm{i}}$, timing differences and derived spatial offsets: $d X=\mathrm{v} . d T / 2$, where v is the stimulus velocity and $d T$ is the shift in the absolute peak-conductance time between the preferred and null directions. A positive value means that the temporal shift was equivalent to activation of inputs ahead of the stimulus during movement in the null direction.

${ }^{a}$ Obtained from a subset of 9 of the 16 cells in which $g_{\mathrm{i}}$ during preferred-direction stimuli displayed a peak early in the response.

${ }^{b}$ The outlier off-response point (Fig. $5 C$ ) is not included in this average. The average including this point is $1.54 \pm 0.73$.
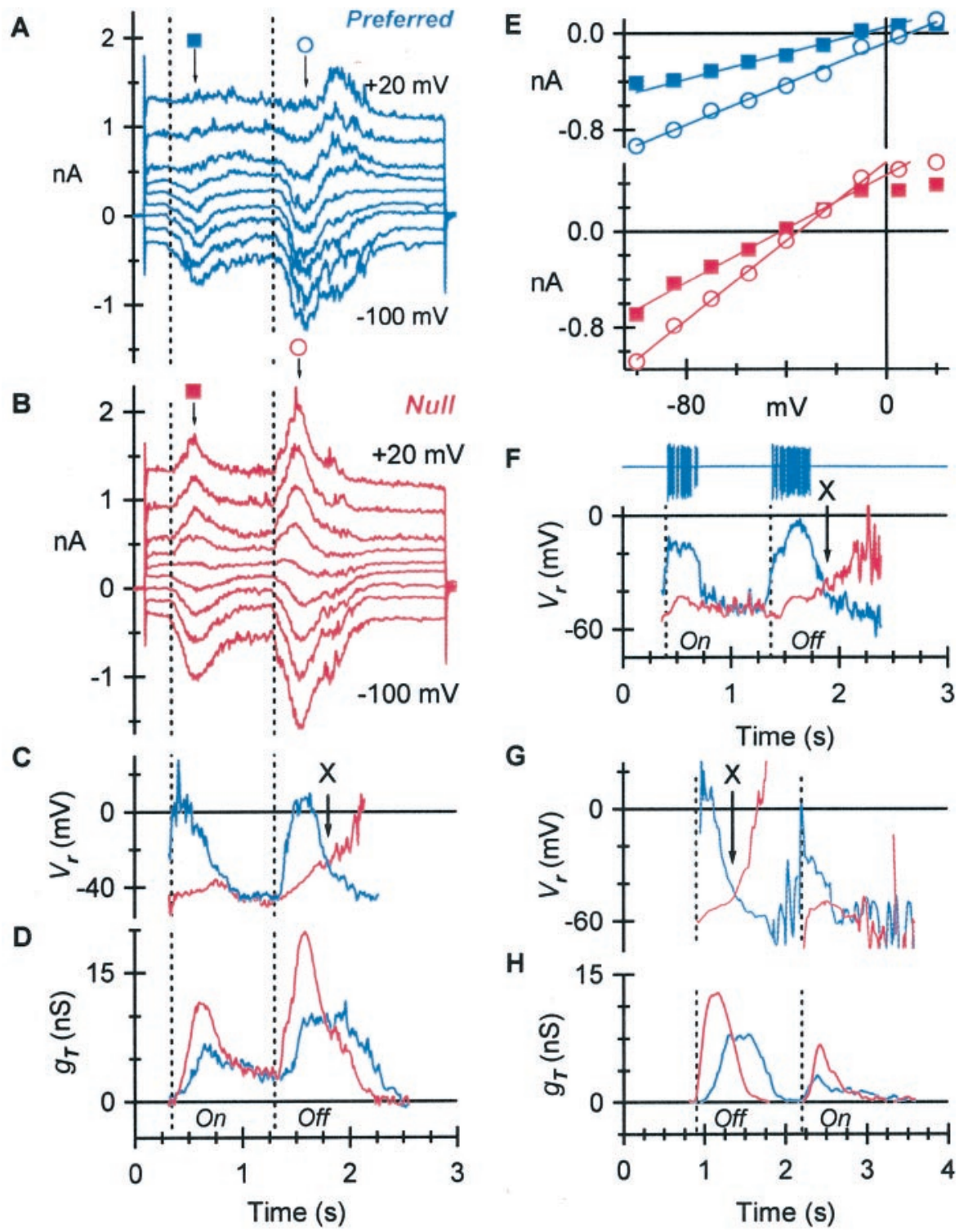

Figure 2. Analysis of the membrane conductance during motion along the preferred-null axis for a single stimulus. Blue data represent preferred direction; red data represent null direction. $A, B$, Synaptic currents elicited by a bright bar moving in the preferred and null directions at a series of holding potentials starting at $-100 \mathrm{mV}$ and incrementing by $15 \mathrm{mV}$ to $+20 \mathrm{mV}$. The broken vertical lines delineate the start of the leading- and trailingedge responses. Because the stimulus bar was brighter than the background, the leading-edge response is an on-response, and the trailing-edge response is an off-response. The symbols above the records show the time points for measuring the current-voltage relations shown in E. The solid lines show examples of linear fits used to determine the synaptic reversal potential $V_{r}$ and the synaptic conductance $g_{T}$. C, $D, I-V$ relations were measured at $10 \mathrm{msec}$ intervals to plot out the time dependence of these parameters. $F, V_{r}$ in another cell showing the relative timing of extracellular action potentials during preferred-direction motion and recorded before applying the patch-clamp electrode. $G, H, V_{r}$ and $g_{T}$ recorded during negativecontrast stimuli showed characteristics similar to those for positive contrasts.

of the on-response, which superimposed during the late phase of the response, indicating that the synaptic currents were not direction selective at these times. These distinct characteristics suggest that there are basic differences in the synaptic mechanisms underlying direction selectivity for the on- and off-responses.

\section{Synaptic conductance}

The peak conductance was larger in the null direction than the preferred direction (Fig. 2D,H). This would be compatible with a presynaptic model of direction selectivity in which inhibition was larger in the null direction, with no change in the time course 


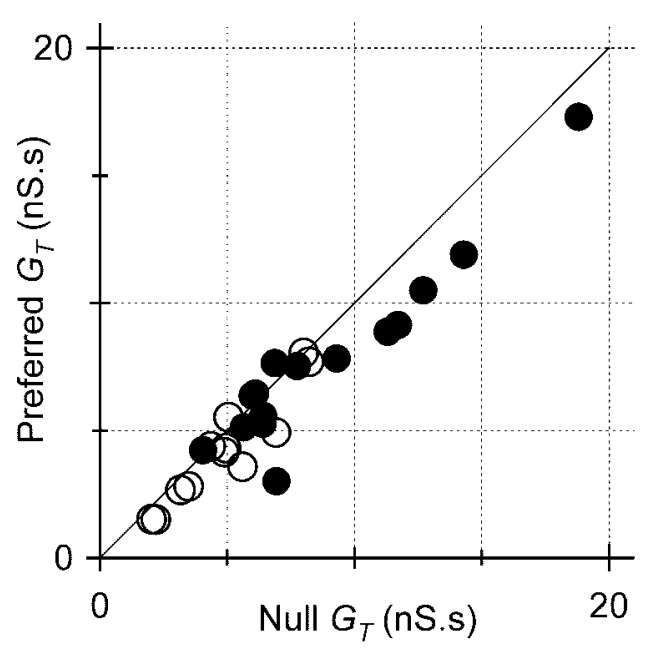

Figure 3. Integrated conductance. Each point shows a single measurement of the total integrated conductance, $G_{T}$. The solid symbols show averages of one to three determinations in the 16 cells that were analyzed further. The open symbols show the average of one to three measurements in 12 additional cells.

of the inputs. However, a greater peak conductance in the null direction would also be compatible with a postsynaptic model in which the closer temporal correlation of inhibition and excitation in the null direction results in greater summation of the inhibitory and excitatory conductances, with no change in their relative magnitudes.

The off-response may satisfy the latter possibility. Although the peak off-conductance was smaller in the preferred direction, the waveform was consistently broader (Fig. 2D, H, blue line). Thus it is possible that the same total conductance is activated, but with differing temporal dispersion. In contrast, the on-response appeared to have a very similar time course in the preferred and null directions, more in line with a presynaptic mechanism. The reversal potential trajectories support this interpretation. During the on-response, the reversal potential in the null direction was essentially constant, indicating that excitation and inhibition had very similar time courses (Eq. 2). During the off-response, the reversal potential in the null direction changed continuously, consistent with different spatiotemporal characteristics for the two inputs, as expected for a postsynaptic mechanism.

For a purely postsynaptic model, the total synaptic conductance should be equal in the preferred and null directions, whereas for presynaptic mechanisms, there should be an imbalance. We examined this quantitatively by integrating the conductance records in the two directions. When the preferred $G_{T}$ is plotted against the null $G_{T}$ (Fig. 3), many of the points lie below the unity line, indicating that the integrated conductance was slightly larger in the null direction, averaging $118 \pm 23 \%$ for 28 DSGCs. Although the difference was small and not shown by some cells, it suggested that there might be presynaptic components to the directional mechanism.

\section{Excitatory and inhibitory components}

To identify whether the asymmetry lies in the excitatory or inhibitory inputs to the ganglion cells, we resolved their separate contributions to the conductance records. Similar to previous electrophysiological studies, we found that the on-response of a DSGC could be quite different in size from the off-response, with the off-responses tending to be larger. To make a meaningful comparison between the on- and off-responses, cells with markedly different responses were excluded from the analysis, leaving a subset of 16 cells with broadly comparable responses (Fig. 3, O). For these analyzed cells, the $G_{T}$ of the on-responses in both directions ranged from 21 to $131 \%$ of the $G_{T}$ of the off-responses $($ mean $=49 \pm 26 \%)$.

To separate the conductance records into excitatory and inhibitory components (Eq. 3 and 4), we made the following assumptions (see Materials and Methods). (1) The DSGC is isopotential; theoretical results from Koch et al. (1990) indicate that this approximation will result in errors in the magnitude but not the ratio of the conductances (see Discussion). (2) Synaptic and dendritic membrane conductances are linear. (3) There are only two synaptic conductances contributing to the responses: excitation and inhibition. The reversal potentials for excitation and inhibition were assigned for each cell as described in Materials and Methods. In the sample of 16 cells, $V_{e}=+4.3 \pm 5.5 \mathrm{mV}$ with a range 0 to $+20 \mathrm{mV}$, and $V_{i}=-65.7 \pm 2.3 \mathrm{mV}$ with a range -65 to $-75 \mathrm{mV}$. (4) The excitatory and inhibitory conductances are greater than or equal to zero.

The calculated excitatory and inhibitory components provide a rationale to account for the time-dependence of $V_{r}$ and $g_{T}$. The qualitative features described here were similar for all cells, although there were marked quantitative differences from cell to cell. The peak amplitude of the excitatory conductance, $g_{e}$, tended to be larger in the preferred direction, but the overall time course was independent of direction for both the on- and off-responses (Fig. $4 A, E$ ). By contrast, the time course of the inhibitory conductance, $g_{i}$, differed dramatically in the preferred and null directions (Fig. 4B,F).

For the off-response, the inhibition in the preferred direction was significantly delayed compared with the null direction, consistent with an asymmetric spatial offset (Fig. 4B, $F$ ). For the on-response, a distinction must be made between positive- and negative-contrast stimuli. During negative-contrast stimuli, the inhibition was smaller in the preferred direction but its time course was unchanged, suggesting that presynaptic mechanisms control this input (Fig. 4B). During positive-contrast stimuli, inhibition comprised an early transient phase (Fig. $4 F$, delineated by the solid line beneath the trace) followed by a sustained phase (Fig. $4 F$, delineated by the broken line beneath the trace). The magnitude of the early phase was strongly modulated in opposite directions, also consistent with presynaptic direction-selective mechanisms. The late phase of the on-response was nondirectional and superimposed for opposite-direction stimuli. Moreover, synaptic currents active during this late phase did not generate spikes in either direction (Fig. $2 F$ ). In contrast, a sustained phase was not present during on-responses elicited during negative-contrast stimuli (Fig. 4B), and it was clear during such responses that, unlike the off-responses, the inhibition is not delayed in the preferred direction. Although it is more difficult to discern during positive-contrast stimuli, the on-responses appeared to lack any inhibitory delay in the preferred direction because, as noted above, the late phase of the on-response was nondirectional (Fig. $4 F$ ).

The reversal potential trajectories can be understood in terms of the relative trajectories of the excitatory and inhibitory conductances in each direction. In the preferred direction, excitation dominated because the inhibition was either reduced (onresponse) or delayed (off-response) (Fig. 4C,G). In the null direction, $V_{r}$ was essentially constant during the on-response because the excitation and inhibition had similar time courses (Fig. 

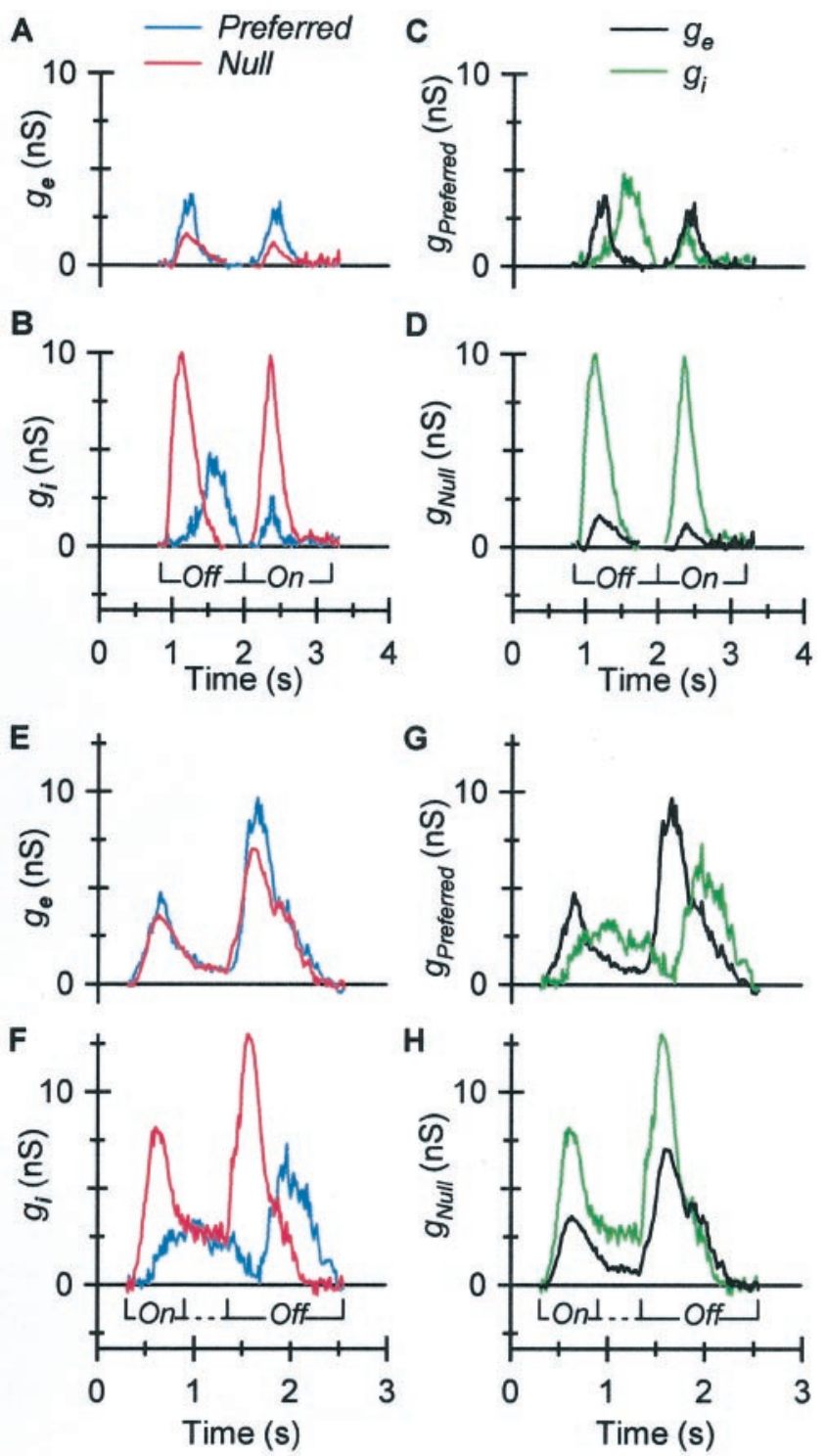

Figure 4. Separation of $g_{T}$ into $g_{e}$ and $g_{i}$. Each trace is the response to a single stimulus. Comparison of the time course of $g_{e}(A, E)$ and $g_{i}(B, F)$ for positive $(A, B)$ and negative $(E, F)$ contrast stimuli. $C, D, G, H$, The same records replotted to compare preferred- and null-direction responses. The solid lines under the records delineate the intervals used to calculate the direction-selective integrals in Figure 5.

$4 D, H)$. By contrast, $V_{r}$ tended to increase during the off-response, leading to the characteristic crossover of the $V_{r}$ trajectories. The crossover resulted from a fall in the ratio of inhibition to excitation late in the response, attributable to an earlier onset and a narrowing of the inhibitory component. In summary, onresponses appeared to be dominated by a directional inhibitory input, whereas off-responses displayed both a directional inhibitory input and a change in temporal correlation as expected for a postsynaptic mechanism.

The inhibition during the on-responses was invariably larger in the null direction, but it is not clear whether other factors contribute to the imbalance in the total conductance shown in Figure 3. Therefore, we integrated the two conductance components, $g_{\mathrm{e}}$ and $g_{\mathrm{i}}$, separately for the on- and off-responses. The integrals for the on-responses during positive-contrast stimuli were calculated over the transient phase (see above), delineated by the solid lines
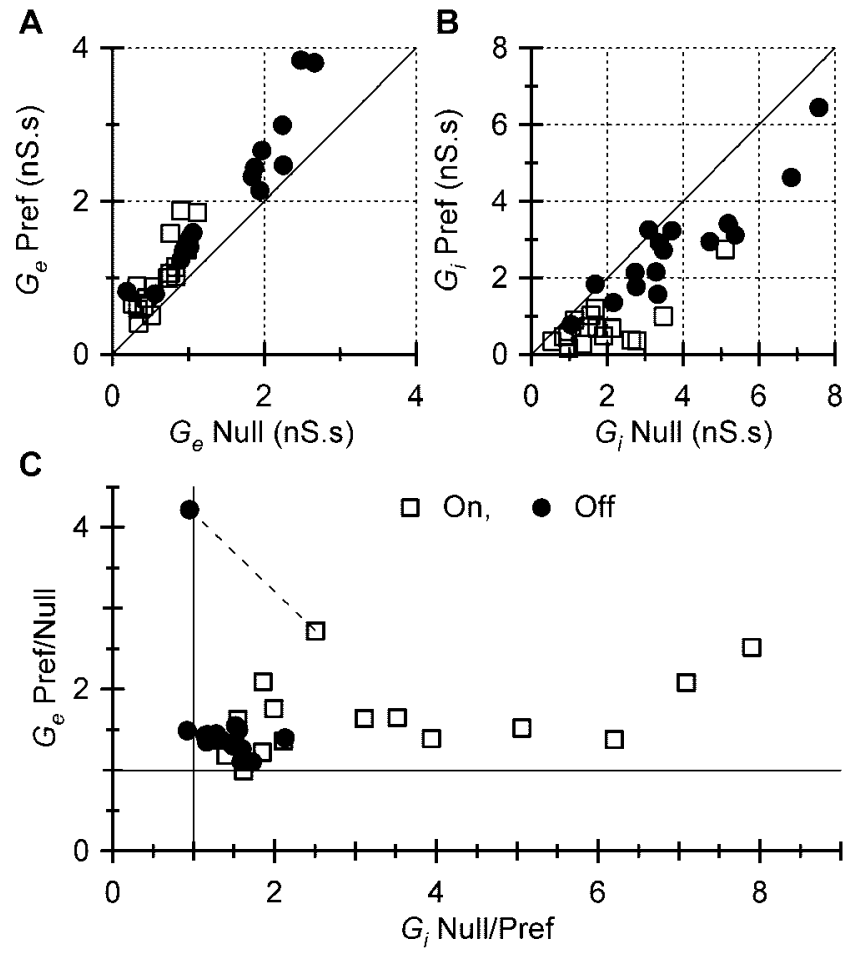

Figure 5. Integrals of the conductance components for the on-response $(A, \square)$ and the off-response $(B, \bullet)$. Note the change in scale between $A$ and $B$. The unity slope line shows the expectation for equal responses in the preferred and null directions. $C$, Conductance ratios obtained from the data in $A$ and $B$; the on-off pair for an outlier is connected by a dashed line.

beneath the records in Figure $4 F$, thereby avoiding the sustained nondirectional inhibition active late in the on-response. Inclusion of this nondirectional component during the analysis only reduced the estimated asymmetry of the on-inhibition.

The excitatory component was larger in the preferred direction (Fig. $5 A$ ), and on average, this presynaptic asymmetry was similar for the on- and off-responses (Table 1). Similarly, the inhibitory component was consistently larger in the null direction (Fig. 5B). These differences are illustrated more clearly when the preferred/ null $G_{\mathrm{e}}$ ratio is plotted against the null/preferred $G_{\mathrm{i}}$ ratio (Fig. $5 C$ ). For a purely postsynaptic scheme, the points should cluster around the intersection of the unity lines. For the great majority of points, both the $G_{\mathrm{e}}$ ratio and the $G_{\mathrm{i}}$ ratio were greater than unity, indicating that the cells received both directional excitation and directional inhibition, consistent with their preferred direction. The off-responses clustered nearer the intersection than the on-responses, indicating that the presynaptic mechanisms were more potent for the on-responses. The mean values of the $G_{\mathrm{e}}$ and $G_{\mathrm{i}}$ ratios for the on- and off-responses are shown in Table 1, which also shows the preferred-null ratio of the total directional conductance, $G_{\mathrm{D}}$, defined as the sum of $G_{\mathrm{e}}$ and $G_{\mathrm{i}}$ evaluated over the "On" and "Off" intervals delineated in Figure 4.

For the off-responses, the directional excitation and directional inhibition were of equal magnitude, and thus the total offconductance was balanced for the preferred and null directions. For the on-responses, directional inhibition tended to be stronger, and thus the total on-conductance was greater in the null direction. The imbalance observed in Figure 3 for the integrated conductance can be attributed, therefore, mainly to the onresponse, diluted by the larger off-response, and by inclusion of 


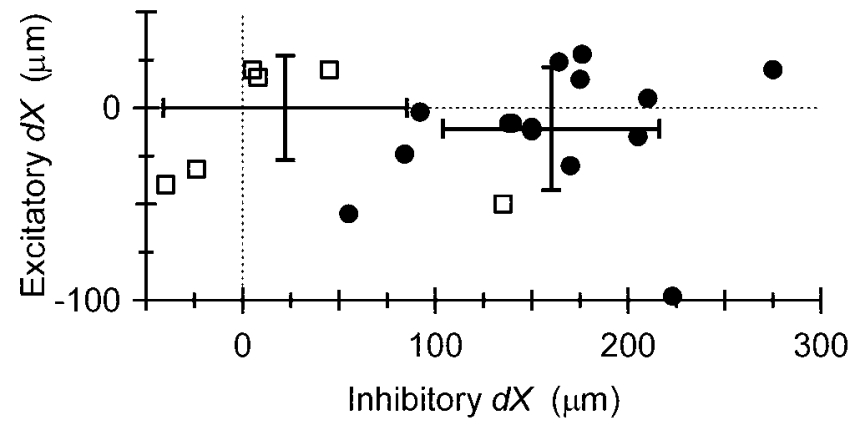

Figure 6. The off-inhibitory conductance is offset in the null direction. The symbols show the inferred spatial offset, $d X$, calculated from the shift in the peak-conductance time observed for preferred- and null-direction motion. A positive spatial offset means that the conductance was delayed during preferred-direction motion relative to null-direction motion. $\square$, On-responses; $\boldsymbol{Q}$, off-responses. The on-response data are a subset of 9 of the 16 cells in which the preferred $g_{i}$ displayed a clear peak early in the response. Also shown are the mean and SDs for the groups of points. Only the inhibitory off-conductance is significantly different from zero.

the nondirectional conductance active during the late phase of the on-response. The broad range of ratios in Figure $5 C$ is noteworthy, indicating that the cells can be direction selective when they receive nondirectional excitation or nondirectional inhibition.

The extent of the spatial offset of the inhibition was estimated from the timing differences of the inhibitory peaks. The calculations assume that the receptive field is centered with respect to the stimulus (which is aligned to the soma) and that the synaptic delays are the same in the null and preferred directions. Consistent with this assumption, the excitatory conductance peaks were coincident in the preferred and null directions (Fig. 4A,E). The absolute peak-conductance time, relative to the start of a stimulus trial, was measured for excitation and inhibition in the preferred and null directions. The difference, $d T$, preferred minus null was converted into an equivalent spatial offset; for a stimulus speed of $\nu \mu \mathrm{m} / \mathrm{sec}$, the spatial offset was estimated as $d X=\nu \cdot d T / 2$. The only spatial offset that diverged significantly from zero was that for the inhibition during the off-response, for which $d X=160 \pm$ $56 \mu \mathrm{m}$ (Fig. 6, Table 1). Because the excitatory conductance peaks were coincident in the null and preferred directions, the spatial offset of the inhibition was also estimated from the timing differences between the excitation and inhibition in a given direction. This does not assume that the receptive field is centered relative to the stimulus. The derived spatial offset for the inhibition during the off-response in the null direction averaged $148 \pm 48 \mu \mathrm{m}$, in close agreement with the figure above.

\section{High chloride}

Previously, we showed that the inhibition acts directly on the dendrites of the DSGC, and we argued that the direction selectivity is generated postsynaptically (Taylor et al., 2000). The key evidence was the apparent loss of directional responses observed at $-30 \mathrm{mV}$ when the patch electrode contained a high chloride concentration (Fig. 7A,C). The conductance analysis provides further insights into the effects of high intracellular chloride (Fig. $7 B, D)$. At the commencement of patch recording, the $V_{\mathrm{r}}$ trajectory was similar to that observed in control cells (Fig. $7 B$ ). Approximately $7.5 \mathrm{~min}$ later, $V_{\mathrm{r}}(t)$ had shifted to more positive potentials, with little change in the conductance (Fig. 7D), and the crossover was still evident during the late phase of the off-

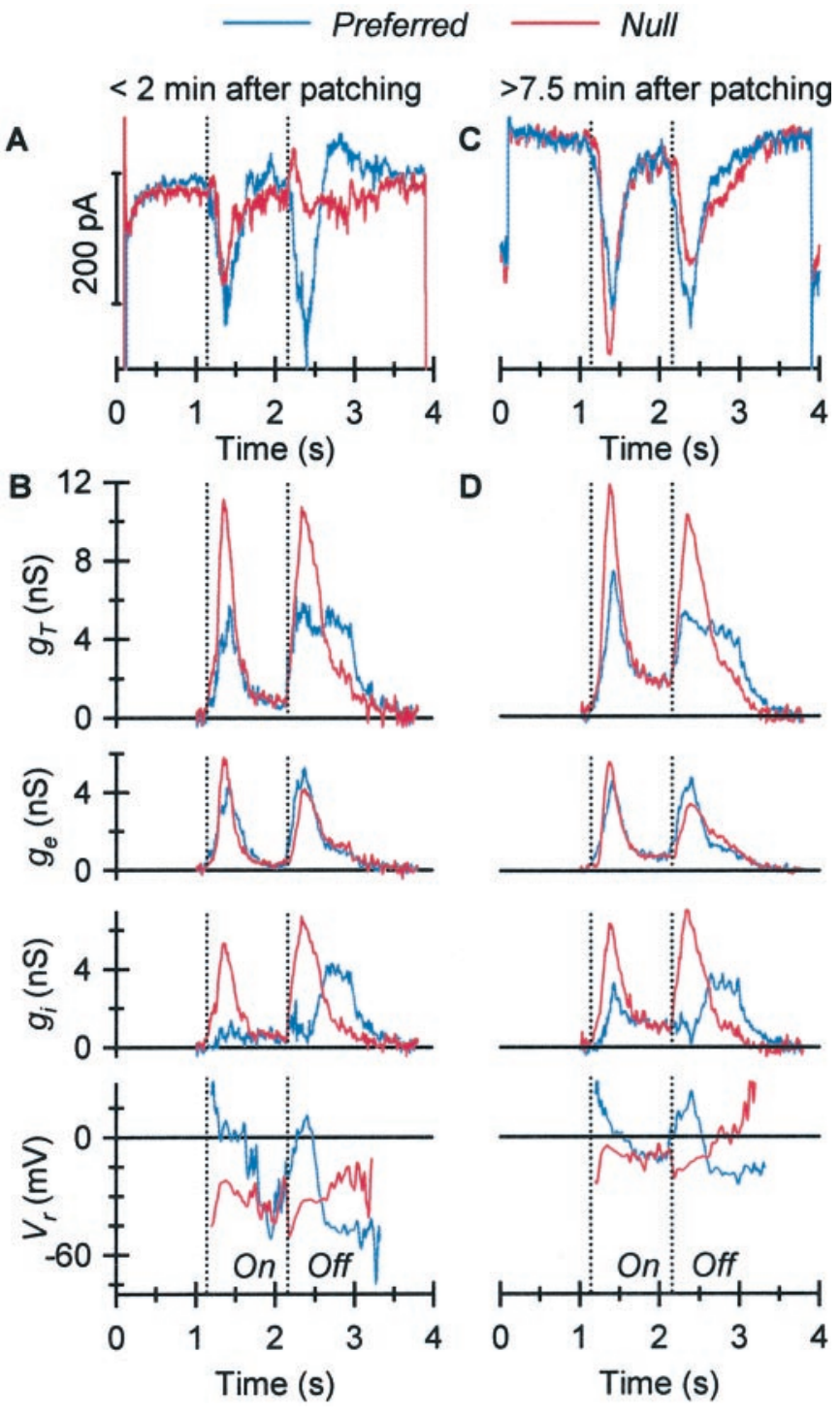

Figure 7. Effect of high intracellular chloride. $A, B$, Records in response to preferred (blue) and null (red) stimuli at the commencement of the whole-cell recording. $C, D$, Similar data obtained 7.5 min later. The shift in $V_{i}$ is evident, but the overall time course and magnitude of the conductances are unchanged.

response. The main effect of the high chloride appeared to be a shift in $V_{\mathrm{i}}$. In five cells, $V_{\mathrm{i}}$ and $V_{\mathrm{e}}$ were initially $-63 \pm 7 \mathrm{mV}$ and $+8 \pm 4 \mathrm{mV}$, but 2-6 min later, $V_{\mathrm{i}}$ and $V_{\mathrm{e}}$ were set to $-28 \pm 5$ and $18 \pm 11 \mathrm{mV}$ to satisfy assumption (4) above. The high chloride did not change the magnitude of the excitatory and inhibitory conductances that impinge on the DSGC; thus, the total integrated conductance did not change significantly from the initial value. Under physiological conditions, the positive reversal potential for inhibition tends to generate spikes in the null direction, thus reducing direction selectivity.

\section{DISCUSSION}

\section{Locus of direction selectivity}

The results reported here indicate that direction selectivity is generated by the combination of three distinct synaptic asymmetries: (1) a presynaptic mechanism producing stronger excitation in the preferred direction, (2) a presynaptic mechanism produc- 
ing stronger inhibition in the null direction, and (3) postsynaptic interaction of the excitation with spatially offset inhibition. The three mechanisms were not uniformly expressed by the on- and off-responses, although the extracellularly recorded responses were very similar. The on-responses appeared to rely on presynaptic mechanisms. The off-responses were more consistent with a mix of presynaptic and postsynaptic mechanisms.

For both the on- and off-responses, the precise mix of presynaptic and postsynaptic mechanisms was quite variable, and in some cells one or another of the presynaptic components was absent (points close to the unity lines in Fig. 5). This variability raises the possibility that DSGCs are programmed to generate a specific functional phenotype during development but that there is considerable latitude in the eventual mix of synaptic mechanisms used to achieve this goal. The large variability in the contribution from each of the three mechanisms, both among different cells and between the on- and off-responses, suggests that no single mechanism is essential for the generation of direction selectivity. Moreover, the mechanistic variability argues against the idea that different mechanisms are required to process different types of visual stimuli (Grzywacz et al., 1998). Thus, the robustness of the directional trigger feature over a wide range of stimulus conditions (Barlow and Levick, 1965; Wyatt and Daw, 1975) probably reflects robustness in the individual synaptic mechanisms.

A postsynaptic mechanism reliant on shunting inhibition requires that the synapses be electrotonically remote from the soma for direction selectivity to be generated locally within the dendritic tree (Torre and Poggio, 1978; Koch et al., 1983). The linearity of the $I-V$ relations and the clear reversal potentials observed at positive potentials seem inconsistent with an electrotonically extensive dendritic arbor. However, the dendritic arbors were probably more electrotonically compact during the recordings shown here, because the intracellular solution contained cesium ions rather than potassium ions. Further experiments will need to be performed using potassium-based intracellular solutions to determine whether the off-arbors really are electrotonically extensive. The off-dendritic arbor has a greater density of thin terminal dendrites than the on-dendritic arbor and is located more distally from the soma (Vaney, 1984; Oyster et al., 1993). Both of these factors could favor a more electrotonically extensive arbor. Moreover, the off-dendrites can arise from ondendrites of any branching order, and thus any postsynaptic mechanism operating within the on-arbor may inappropriately shunt excitation being transmitted from the off-arbor.

The conductance analysis revealed that both the excitatory and inhibitory inputs to DSGCs may be tuned directionally and that the response bias is consistent with the preferred direction of the cells. In an earlier study we argued for a simpler scheme involving only postsynaptic mechanisms (Taylor et al., 2000). Yet the study reported here presents new evidence for presynaptic mechanisms. How can this be reconciled with the previous findings? The earlier study attempted to account for the behavior of the DSGCs by the most parsimonious interpretation, which involved testing the data against each possible mechanism rather than combinations of mechanisms. In the first experiment, depolarization of the DSGCs from -70 to $-30 \mathrm{mV}$ increased the direction selectivity of the cell, providing evidence against the presynaptic excitatory mechanism. In the second experiment, raising the inhibitory reversal potential with high intracellular chloride mostly abolished the direction selectivity, consistent with a postsynaptic mechanism. If the response had relied on a presyn- aptic mechanism, then the high chloride should have either reversed the direction selectivity (for a directional inhibitory input) or not affected the direction selectivity (for a directional excitatory input). The parsimonious conclusion of Taylor et al. (2000), that there appeared to be no asymmetry in the synaptic inputs to the DSGCs, is consistent with the present finding that the total conductance is almost equal for the preferred and null directions (Fig. 3). This balance of the conductance in the preferred and null directions is attributable partly to the complementary balance of directional excitation and directional inhibition and partly to the tendency for off-responses to be larger, a factor that was not controlled for previously.

\section{Errors in conductance estimates in a non-isopotential neuron}

To separate the conductance records into excitatory and inhibitory components, we assumed that the DSGCs were isopotential (Anderson et al., 2000; Borg-Graham, 2001). However, a simple test confirmed the expectation that this is an approximation. During a small voltage step, the capacitive transient in the DSGCs required a sum of exponentials to be fitted accurately (data not shown), demonstrating that these cells are not isopotential. In a non-isopotential cell, a point voltage clamp at the soma will not faithfully resolve synaptic currents impinging on the dendritic arbor. Because the inputs become more electrically distant, their visibility to an electrode at the soma deteriorates.

Koch et al. (1990) showed that the actual conductance at the dendritic site is always larger than the conductance measured at the soma but that this error is independent of the synaptic reversal potential, even for mixed excitatory and inhibitory inputs. Thus, even if there are nonlinear shunting interactions between inhibition and excitation at some given potential, this will not affect the estimate of the conductance ratios of the two inputs determined over a range of potentials. The theoretical approach used by Koch et al. (1990) assumed that the time-constants of the synaptic inputs are long compared with the time-constants for the decay of voltage transients within the dendritic arbor. This requirement is adequately satisfied in the present case. The capacitive transients at the onset and termination of a voltage step are barely visible in the records shown in Figure 2 because they decayed rapidly compared with the time course of the lightevoked synaptic currents.

Separation of the conductance into excitatory and inhibitory components also required estimates for the reversal potentials of the two inputs. These were not measured directly and therefore were set to reasonable values: $0 \mathrm{mV}$ for excitation and $-65 \mathrm{mV}$ for inhibition. Adjustment was sometimes required to ensure that both conductance components remained positive. Changing either $V_{e}$ or $V_{i}$ will change the relative magnitude of the associated conductance (Eq. 3 and 4). Thus, uncertainty in the reversal potentials implies uncertainty in the magnitude of the conductances. However, this uncertainty does not affect the conclusions from the data based on the ratios of the conductances, because the same reversal potentials were used for analysis in the preferred and null directions.

The estimation of the presynaptic components relies on the ratio of conductances measured in the preferred versus null directions. Because the total light-evoked conductance was essentially constant in the preferred and null directions (Fig. 3), the conductance errors will be independent of stimulus direction. The conductance errors for the off-responses, however, are complicated by the delay in the inhibitory conductance. The temporal 
offset of inhibition relative to excitation in the preferred direction will tend to reduce the conductance errors of both inputs, whereas the temporal correlation of the inputs in the null direction will tend to increase the errors. Thus, the excitatory ratio will tend to be overestimated, and the inhibitory ratio will tend to be underestimated. Without quantitative modeling, it is not possible to say how large these errors in estimation might be, but they are probably not large, because the observed conductance ratios were not correlated with the magnitude of the total conductance.

\section{Implications for synaptic circuitry}

Our results indicate that direction selectivity for both on- and off-responses is generated to some extent by a push-pull mechanism, where complementary changes in excitation and inhibition drive the cell to a greater or lesser degree with only small changes in overall membrane conductance (Watanabe and Murakami, 1984; Anderson et al., 2000). However, although this study characterized the synaptic inputs to the DSGCs in unprecedented detail, our results raise many questions. For example, the results do not reveal whether the stronger excitation in the preferred direction arises from preferred-direction facilitation or from nulldirection inhibition acting presynaptically on the excitatory inputs. Nor do the results tell us whether both the glutamatergic input from cone bipolar cells and the cholinergic input from starburst amacrine cells are directional and, if so, whether similar synaptic mechanisms are responsible for their direction selectivity. For example, it has been proposed that the terminal synapses on the radial dendrites of starburst amacrine cells will be more strongly activated by centrifugal stimulation than by centripetal stimulation (Borg-Graham and Grzywacz, 1992), but such a structural mechanism is unlikely to apply to the compact axon terminal of a bipolar cell.

Many of the neuronal models of direction selectivity that have been put forward invoke a key role for starburst cells, which are two mirror-symmetric populations of amacrine cells that cofasciculate with the bistratified DSGCs (Vaney et al., 1989; Vaney and Pow, 2000). These amacrine cells contain both acetylcholine and GABA and thus could contribute to both the excitatory and inhibitory mechanisms (Vaney et al., 1989; Borg-Graham and Grzywacz, 1992; Grzywacz et al., 1997). The role of starburst cells within the direction-selective circuit is controversial, however, with one report claiming that they are critical (Yoshida et al., 2001) and another claiming that they are not essential (He and Masland, 1997). Available evidence indicates that the two populations of starburst cells are structurally and functionally equivalent (except for the sign of their responses) (Bloomfield, 1992), and therefore they may underlie the presynaptic mechanisms that are common in the on- and off-responses. In contrast, a different type of amacrine cell branching only in the off-sublamina may mediate the spatially offset inhibition that distinguishes the offresponses from the on-responses. A change in the timing of the off-inhibition in the preferred and null directions predicts a spatial offset of $\sim 160 \mu \mathrm{m}$, which is approximately half the dendritic diameter of a starburst cell in mid-peripheral retina (Tauchi and Masland, 1984; Vaney, 1984).

Our results show that as far as the spike output is concerned, the on- and off-dendritic arbors of a DSGC perform essentially the same computation but use different combinations of synaptic mechanisms. The challenge for future research is to identify the synaptic circuitry that generates the directional signals, both presynaptically in the excitatory and inhibitory neurons and postsynaptically in the DSGCs. This is the same challenge that faced Barlow et al. $(1964,1965)$ almost 40 years ago, when they first characterized the DSGCs in rabbit retina; however, we now know that the synaptic mechanisms underlying direction selectivity are more diverse than they had envisaged.

\section{REFERENCES}

Amthor F, Grzywacz N (1991) Nonlinearity of the inhibition underlying retinal directional selectivity. Vis Neurosci 6:197-206.

Anderson JS, Carandini M, Ferster D (2000) Orientation tuning of input conductance, excitation, and inhibition in cat primary visual cortex. J Neurophysiol 84:909-926.

Ariel M, Daw NW (1982) Pharmacological analysis of directionally sensitive rabbit retinal ganglion cells. J Physiol (Lond) 324:161-185.

Barlow H, Levick W (1965) The mechanism of directionally selective units in rabbit's retina. J Physiol (Lond) 178:477-504.

Barlow H, Hill R, Levick W (1964) Rabbit retinal ganglion cells responding selectively to direction and speed of image motion in the rabbit. J Physiol (Lond) 173:377-407.

Bloomfield SA (1992) Relationship between receptive field and dendritic field size of amacrine cells in the rabbit retina. J Neurophysiol 68:711-725.

Borg-Graham L (2001) The computation of directional selectivity in the retina occurs presynaptic to the ganglion cell. Nat Neurosci 4:176-183.

Borg-Graham LJ, Grzywacz NM (1992) A model of the directional selectivity circuit in retina: transformations by neurons singly and in concert. In: Single neuron computation, pp 347-375. Boston: Academic.

Grzywacz NM, Tootle JS, Amthor FR (1997) Is the input to a GABAergic or cholinergic synapse the sole asymmetry in rabbit's retinal directional selectivity? Vis Neurosci 14:39-54.

Grzywacz NM, Amthor FR, Merwine DK (1998) Necessity of acetylcholine for retinal directionally selective responses to drifting gratings in rabbit. 512:575-581.

He S, Masland RH (1997) Retinal direction selectivity after targeted laser ablation of starburst amacrine cells. Nature 389:378-382.

Kittila C, Massey S (1997) Pharmacology of directionally selective ganglion cells in the rabbit retina. J Neurophysiol 77:675-689.

Koch C, Poggio T, Torre V (1983) Nonlinear interactions in a dendritic tree: localization, timing, and role in information processing. Proc Natl Acad Sci USA 80:2799-2802.

Koch C, Douglas R, Wehmeier U (1990) Visibility of synaptically induced conductance changes: theory and simulations of anatomically characterized cortical pyramidal cells. J Neurosci 10:1728-1744.

Oyster C, Amthor F, Takahashi E (1993) Dendritic architecture of ONOFF direction-selective ganglion cells in the rabbit retina. Vision Res 33:579-608.

Tauchi M, Masland RH (1984) The shape and arrangement of cholinergic neurons in the rabbit retina. Proc R Soc Lond B Biol Sci 223: 101-119.

Taylor WR, He S, Levick WR, Vaney DI (2000) Dendritic computation of direction selectivity by retinal ganglion cells. Science 289:2347-2350.

Torre V, Poggio T (1978) A synaptic mechanism possibly underlying directional selectivity to motion. Proc R Soc Lond B Biol Sci 202:409-416.

Vaney D (1994) Territorial organization of direction-selective ganglion cells in rabbit retina. J Neurosci 14:6301-6316.

Vaney D, He S, Taylor W, Levick W (2001) Direction-selective ganglion cells in the retina. In: Motion vision. Computational, neural and ecological constraints (Zanker J, Zeil J, eds), pp 13-56. Berlin: Springer.

Vaney DI (1984) "Coronate" amacrine cells in the rabbit retina have the "starburst" dendritic morphology. Proc R Soc Lond B Biol Sci 220:501-508.

Vaney DI, Pow DV (2000) The dendritic architecture of the cholinergic plexus in the rabbit retina: selective labeling by glycine accumulation in the presence of sarcosine. J Comp Neurol 421:1-13.

Vaney DI, Collin SP, Young HM (1989) Dendritic relationships between cholinergic amacrine cells and direction-selective ganglion cells. In: Neurobiology of the inner retina (Weiler R, Osborne NN, eds), pp 157-168. Berlin: Springer.

Vardi N, Zhang LL, Payne JA, Sterling P (2000) Evidence that different cation chloride cotransporters in retinal neurons allow opposite responses to GABA. J Neurosci 20:7657-7663.

Watanabe S, Murakami M (1984) Synaptic mechanisms of directional selectivity in ganglion cells of frog retina as revealed by intracellular recordings. Jpn J Physiol 34:497-511.

Wyatt HJ, Daw ND (1975) Directionally sensitive ganglion cells in the rabbit retina: specificity for stimulus direction, size and speed. J Neurophysiol 38:613-626.

Wyatt HJ, Daw ND (1976) Specific effects of neurotransmitter antagonists on ganglion cells in rabbit retina. Science 191:204-205.

Yoshida K, Watanabe D, Ishikane H, Tachibana M, Pastan I, Nakanishi S (2001) A key role of starburst amacrine cells in originating retinal directional selectivity and optokinetic eye movement. Neuron 30:771780 . 